\title{
Thermal-Fluid Flow Transport Phenomenon over Slot-Perforated Flat Plates Placed in Narrow Channel
}

\author{
Shuichi Torii* \\ Kumamoto University, Kumamoto 860-8555, Japan \\ and \\ Wen-Jei Yang $\ddagger$ \\ University of Michigan, Ann Arbor, Michigan 48109 \\ DOI: $\underline{10.2514 / 1.25872}$
}

\begin{abstract}
A numerical study is performed to investigate unsteady, two-dimensional, incompressible laminar flow over both sides of a slot-perforated flat surface, which is placed in a narrow channel. Emphasis is placed on the effect of the blockage factor, that is, the ratio of plate thickness $\delta$ to channel width $W$ on the heat-transfer performance and the velocity and thermal fields. It is found from the study that 1 ) when the slot width is increased, the alternating change in the fluid flow disturbs the thermal boundary layer formed along the plate and induces mixing of the upper and lower streams of the plate downstream from the slot, resulting in an amplification of heat-transfer performance; 2) heat-transfer performance at the rear plate is induced with an increase in slot width and $R e$; and 3) by contrast, heat-transfer performance is attenuated with an increase in the blockage factor, whose effect becomes larger in the lower region of the Reynolds number.
\end{abstract}

\section{Nomenclature}

\section{$B$}

$d$

$h$

$k_{q}$

$L$

$N u$

$\overline{N u}$

$P, \Delta P^{\prime}$

$R e$

$T$

$t$

$U$

$U_{i}$

$u_{m}$

$u_{m}$

W

$x_{i}$

$x, y$

$\alpha$

$\beta$

$\Delta X, \Delta Y$

$\delta$

$\theta$

$\lambda$

$v$

$\rho$

$=$ channel length, $\mathrm{m}$

$=$ slot width, $\mathrm{m}$ $=\left(\lambda[\partial T / \partial y]_{w}\right) /\left(T_{w}-T_{\text {inlet }}\right)$

$=$ blockage factor, $\delta / W$

$=$ length of plate, $\mathrm{m}$

$=$ Reynolds number, $U_{m} \delta / v$

$=$ temperature, $\mathrm{K}$

$=$ time, $\mathrm{s}$

$=$ inlet fluid velocity, $\mathrm{m} / \mathrm{s}$

$=$ channel width, $\mathrm{m}$

$=i$ coordinate, $\mathrm{m}$

$=$ coordinate, $\mathrm{m}$

$=$ thermal diffusivity, $\mathrm{m}^{2} / \mathrm{s}$

$=$ coefficient

$=$ mesh size, $\mathrm{m}$ $\left.T_{\text {inlet }}\right)$

$=$ molecular viscosity, $\mathrm{m}^{2} / \mathrm{s}$

$=$ density, $\mathrm{kg} / \mathrm{m}^{3}$
$=$ local heat-transfer coefficient on the plate, $W / \mathrm{m}^{2} / \mathrm{k}$,

$=$ local Nusselt number on the plate, $h \delta / \lambda$

$=$ time-averaged Nusselt number on the plate

$=$ pressure and dimensionless pressure drop, $=\Delta P /\left(0.5 \rho U_{m}^{2}\right)$, respectively

$=$ velocity component in $x$ direction, $\mathrm{m} / \mathrm{s}$

$=$ velocity component in $i$ directions, $\mathrm{m} / \mathrm{s}$

$=$ velocity component in $y$ direction, $\mathrm{m} / \mathrm{s}$

$=$ plate thickness, $\mathrm{m}$

$=$ dimensionless temperature, $\theta=\left(T-T_{\text {inlet }}\right) /\left(T_{w}-\right.$

$=$ thermal conductivity, $W / \mathrm{m} / k$
Presented as Paper 3596 at the 9th AIAA/ASME Joint Thermophysics and Heat Transfer, San Francisco, CA, 5-8 June 2006; received 14 June 2006; revision received 25 September 2006; accepted for publication 3 October 2006. Copyright (C) 2006 by the American Institute of Aeronautics and Astronautics, Inc. All rights reserved. Copies of this paper may be made for personal or internal use, on condition that the copier pay the $\$ 10.00$ per-copy fee to the Copyright Clearance Center, Inc., 222 Rosewood Drive, Danvers, MA 01923; include the code 0887-8722/07 \$10.00 in correspondence with the CCC.

*Professor, Department of Mechanical System Engineering, 2-39-1 Kurokami, and 158837.

${ }^{\dagger}$ Professor, Department of Mechanical Engineering.
Subscripts

inlet $\quad=$ inlet

$w \quad=$ wall surface of the plate

\section{Introduction}

$\mathbf{T}$ HE heat exchanger is an essential unit in heat extraction and recovery systems. With increasing emphasis on economic and energy saving considerations, a great deal of research effort has been devoted to developing more efficient heat-transfer surfaces. In the design of advanced heat exchangers, louver fins, strip fins, wavy fins, and perforated fins are widely used to achieve high heat-transfer efficiency and compactness. A comparison of performances among these surfaces was made by Wong et al. [1]. Kays [2] reported that perforation results in a substantial increase in heat-transfer performance without introducing a pronounced form drag. Heat exchangers with perforated fins may be made either in small size and light weight as air-cooled condensers or in large size as air-cooled heat exchangers. This viewpoint was supported by some investigators $[\underline{1}, \underline{3}]$.

In general, perforated plates may be employed as an extended surface for internal cooling of turbine blades. They are compact and high in heat-transfer performance induced by boundary layer interruption due to perforations without the salient penalty of the form drag [4-9]. Liang [10] and Liang et al. [11] studied the effects of geometry and arrangement of the perforations on fluid flow behavior and disclosed that the ratio of slot length, in the flow direction, to its thickness plays a very important role in flow behavior. To investigate the effects of the Reynolds number and the ratio of $d$ to $\delta$ on the velocity field, the corresponding numerical analysis was carried out by Torii and Yang $[12,13]$. They reported that the flow pattern between two plates placed in a free stream or two-dimensional channel can be classified into four categories depending on the combination of $R e$ and $\mathrm{d} / \delta$. In particular, at a certain combination of $R e$ and $\mathrm{d} / \delta$, an alternating crossing of flow of the fluid streams takes place through the slot from one side to the other side of the plate in the flow direction, so that the heat-transfer performance from a surface wall of the rear plate is intensified [14]. Amon and Mikic [15] performed numerical investigation of the flow pattern and forced convective heat transfer in slotted channels, in which numerical results are compared with the results with plane-channel flow. They disclosed that over a certain Reynolds number the flow exhibits laminar self-sustained oscillations, which cause significant heat- 
transfer enhancement. Suzuki et al. [16] and Nigen and Amon [17] dealt with thermal-fluid flow transport phenomenon in a plane channel with an inline fin array and in a grooved channel, respectively. They reported that heat-transfer performance is induced due to the self-sustained oscillatory flow. Heat-transfer enhancement by the use of slots in channels with uniformly surface-mounted blocks was investigated by Kim and Anand [18]. They dealt with steady two-dimensional laminar flow and disclosed that cooling performance of slotted channels is improved with an increase in slot size.

Both in industrial heat exchangers and in most experimental rigs, the tubes are placed in channels. The effect of the channel walls is expressed by the blockage factor, which is the ratio of the cylinder diameter to the channel width. A higher blockage factor leads to a higher velocity outside the boundary layer, and to different distributions of pressure and velocity on the cylinder circumferences. Consequently, variations of the shell-cylinder distances lead to change in the local heat transfer. The effect of the blockage factor on heat-transfer performance around the cylinder in the channel was summarized by Zukauskas and Ziugzda [19]. By contrast, the corresponding effect on heat transfer from the perforated plates placed in a channel has not been considered.

The present study investigates unsteady thermal and fluid flow transport phenomena over both sides of a slot-perforated surface, which is placed in the narrow channel. Emphasis is placed on the effect of the blockage factor $k q$ on heat-transfer performance and velocity and thermal fields. In this study the flow is assumed to be two dimensional, to keep the computational time manageable. Threedimensional computations, in general, will require extensive $\mathrm{CPU}$ times to conduct parametric tests such as those conducted here. Thus, the effect of three dimensionality, which arises due to either secondary instabilities or end walls, is not taken into account.

\section{Conservation Equations and Numerical Method}

The present study deals with a forced flow over a single-slotperforated surface heated with constant wall temperature in the channel, which consists of two plain straight plates (i.e., the front and rear plates), of length, $L$, and thickness, $\delta$ aligned in the flow direction with a spacing of $d$. Here two plates are set at $y=W / 2$. Figure 1 depicts the proposed physical model and the coordinate system. The following assumptions are imposed in the formulation of the problem: incompressible, laminar, unsteady flow; constant fluid properties; uniform inlet velocity and uniform inlet fluid temperature; and negligible axial conduction (due to the high Peclet number). Under these assumptions, the simplified governing equations for mass, momentum, and energy read

$$
\begin{gathered}
\frac{\partial U_{i}}{\partial x_{i}}=0 \\
\frac{\partial U_{i}}{\partial t}+U_{j} \frac{\partial U_{i}}{\partial x_{j}}=-\frac{1}{\rho} \frac{\partial P}{\partial x_{i}}+v \frac{\partial}{\partial x_{j}}\left(\frac{\partial U_{i}}{\partial x_{j}}\right)
\end{gathered}
$$

and

$$
\frac{\partial T}{\partial t}+U_{j} \frac{\partial T}{\partial x_{j}}=\alpha \frac{\partial}{\partial x_{j}}\left(\frac{\partial T}{\partial x_{j}}\right)
$$

respectively. Initially, the fluid is quiescent and a uniform temperature at $T=T_{\text {inlet }}$. Then, the plate surface temperature is suddenly changed to $T=T_{w}$. Nonslip condition is employed at the plate surface and the channel walls. At the exit, the boundary conditions for the dependent variables are obtained by setting the first derivatives in the axial direction equal to zero. This is because the same use in other flow conditions is permissible for computational convenience if the outlet boundary is located in a flow region, which is sufficiently far downstream from the region of interest. Notice that the above boundary condition at the exit is strictly valid only when the flow is fully developed.
The discretization method employed in the present study uses a finite difference formulation and the discretized forms of the above governing equations read as

$$
\begin{gathered}
U_{i, j}^{n+1}=U_{i, j}^{n}+\Delta t\left[\frac{1}{\Delta X}\left(P_{i, j}^{n}-P_{i+1, j}^{n}\right)-F U X^{n}\right. \\
\left.-F U Y^{n}+V I S X^{n}\right]
\end{gathered}
$$

$$
V_{i, j}^{n+1}=V_{i, j}^{n}+\Delta t\left[\frac{1}{\Delta Y}\left(P_{i, j}^{n}-P_{i, j+1}^{n}\right)-F V X^{n}-F V Y^{n}+V I S Y^{n}\right]
$$

$$
T_{i, j}^{n+1}=T_{i, j}^{n}+\Delta t\left[-F T X^{n}-F T Y^{n}+V I S T^{n}\right]
$$

The subscript and superscript correspond to the cell location and time level, respectively. A detailed description of the above discretization method and $F U X^{n}, F U Y^{n}, V I S X^{n}, F V X^{n}, F V Y^{n}$, and VISY ${ }^{n}$ in Eqs. (4) and (5), are available in the literature $[20,21]$ and thus are not repeated here. $F T X^{n}, F T Y^{n}$, and $V I S T^{n}$ in Eq. (ㅁ) are expressed as

$$
\begin{aligned}
& F T X^{n}=\frac{1}{4 \Delta X}\left[\left(U_{i, j}^{n}+U_{i, j+1}^{n}\right)\left(T_{i, j}^{n}+T_{i+1, j}^{n}\right)+\beta \mid U_{i, j}^{n}\right. \\
& +U_{i, j+1}^{n} \mid\left(T_{i, j}^{n}-T_{i+1, j}^{n}\right)-\left(U_{i-1, j}^{n}+U_{i-1, j+1}^{n}\right)\left(T_{i, j-1}^{n}+T_{i, j}^{n}\right) \\
& \left.-\beta\left|U_{i-1, j}^{n}+U_{i-1, j+1}^{n}\right|\left(T_{i-1, j}^{n}-T_{i, j}^{n}\right)\right] \\
& F T Y^{n}=\frac{1}{4 \Delta Y}\left[\left(V_{i, j}^{n}+V_{i+1, j}^{n}\right)\left(T_{i, j}^{n}+T_{i, j+1}^{n}\right)+\beta \mid V_{i, j}^{n}\right. \\
& +V_{i+1, j}^{n} \mid\left(T_{i, j}^{n}-T_{i, j+1}^{n}\right)-\left(V_{i, j+1}^{n}+V_{i+1, j-1}^{n}\right)\left(T_{i, j-1}^{n}+T_{i, j}^{n}\right) \\
& \left.-\beta\left|V_{i, j-1}^{n}+V_{i+1, j-1}^{n}\right|\left(T_{i, j-1}^{n}-T_{i, j}^{n}\right)\right] \\
& V I S T^{n}=\alpha\left[\frac{1}{\Delta X^{2}}\left(T_{i+1, j}^{n}-2 T_{i, j}^{n}+T_{i-1, j}^{n}\right)\right. \\
& \left.+\frac{1}{\Delta Y^{2}}\left(T_{i, j+1}^{n}-2 T_{i, j}^{n}+T_{i, j-1}^{n}\right)\right]
\end{aligned}
$$

The system variables $P, U, V$, and $T$ are calculated with a staggered grid proposed by Harlow and Welch [20]. Here, $P_{i, j}^{n}$ is pressure at center of cell $(i, j)$ at time level $n, \overline{U_{i, j}^{n}}$ is $x$-direction velocity at middle of right size of cell $(i, j)$ at time level $n, V_{i, j}^{n}$ is $y$-direction velocity at the middle of the top size of the cell $(i, j)$ at time level $n$, and $T_{i, j}^{n}$ is the temperature at the center of cell $(i, j)$ at time level $n$. The processes involved in completing one calculation cycle at an arbitrary time step are as follows: 1) by solving Eqs. (4-6) for $U, V$, and $T$, subject to the appropriate boundary conditions, the new velocities, which involve the values for the contributing pressure and velocities at the precious time-step, are predicted for the entire mesh to be solved; 2) these velocities are iteratively adjusted to satisfy the continuity equation (1) by making appropriate changes in the cell pressures, that is, each cell in the iteration is considered successively and is given a pressure change $\delta p$ that drives its instantaneous velocity divergence to zero [22];

$$
\delta P=-\frac{(1 / \Delta X)\left(U_{i, j}-U_{i-1, j}\right)+(1 / \Delta Y)\left(V_{i, j}-V_{i, j-1}\right)}{\left|2 \Delta t\left[\left(1 / \Delta X^{2}\right)+\left(1 / \Delta Y^{2}\right)\right]\right|}
$$

3) when convergence has been reached with a maximum relative change in the values between successive iterations of less $10^{-4}$, that is, $(1 / \Delta X)\left(U_{i, j}-U_{i-1, j}\right)+(1 / \Delta Y)\left(V_{i, j}-V_{i, j-1}\right) \leq 10^{-4}$, the velocity and pressure fields are determined at the advanced time level and are used as starting values at next time step. These processes are repeated until $t$ reaches a prescribed time, say, $T=120 \mathrm{~s}$ after the calculation is initiated. Note that at this time, the thermal transport 


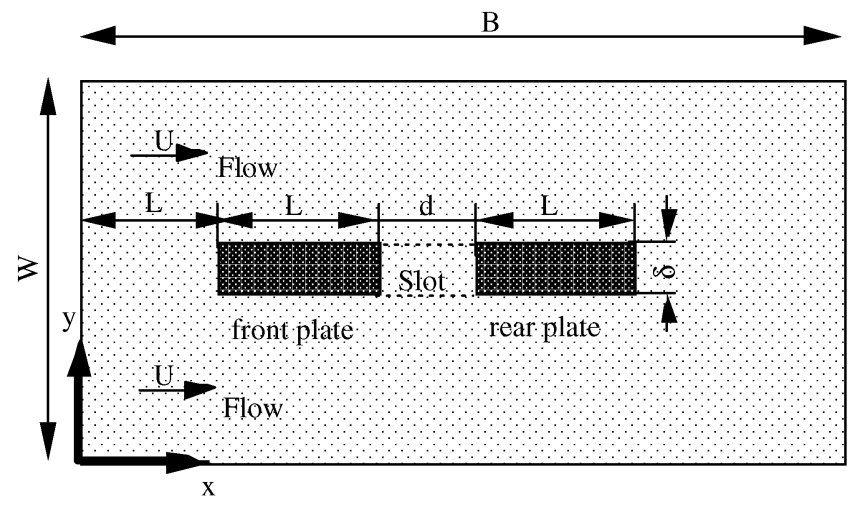

Fig. 1 A schematic of physical system and coordinate.

phenomenon becomes periodic, that is, a steady time-periodic solution is obtained, as shown in the following.

The grid system is changed from $200 \times 400$ to $400 \times 800$ to obtain a grid-independent solution. Numerical computation was performed on a personal computer at time interval $\Delta T=0.00001 \mathrm{~s}$. Here, the maximum time interval employed is determined to ensure the numerical stability. Based on the dataset obtained here, visualization of the flow and thermal fields is carried out using a commercially available 2-D graphics software tool.

In general, the heat transport phenomenon in a channel flow is affected by three dimensionless parameters, that is, $\operatorname{Re}\left(=U_{m} \delta / \nu\right)$, $\operatorname{Pr}$, and $k_{q}$. In the present study, $L / \delta$ is fixed at 2 , and water is used as the working fluid $(P r=0.71)$, whereas $R e$ is ranged from 100 to 1200 , and $k_{q}$ is varied from 0.05 to $0.50 . \mathrm{d} / \delta$ is fixed at 3.0. Note that for $\mathrm{d} / \delta=3.0$, the alternating change in the fluid flow disturbs the thermal boundary layer formed along the plate and induces mixing of the upper and lower streams of the plate downstream from the slot, resulting in an amplification of heat-transfer performance [23].

Simulations with grids of various degrees of coarseness, as mentioned earlier, were conducted to determine the required resolution for grid-independent solutions. Throughout the Reynolds number range considered here, the maximum relative error was estimated to be about $2 \%$ by comparing the solutions on regular and fine grids with twice the grid points. Although a few solutions were computed with half the time step to ensure consistency and time-step independence, there was no substantial discrepancy between two different time intervals.

\section{Results and Discussion}

The structure of the flow over the single-slot-perforated plate and the wake of the rear plate are discussed through the use of computed instantaneous velocity vectors. Figure 2 , for $R e=120$ and $T=100 \mathrm{~s}$, depicts the instantaneous maps of fluctuating velocity vector for the different blockage factor. Notice that velocity components are normalized by dividing by inlet velocity. Figures $2 \mathrm{a}-2 \mathrm{c}$ show the numerical results at $k_{q}=0.1, k_{q}=0.2$, and $k_{q}=\overline{0.5}$, respectively. The corresponding temperature distributions, that is, isotherms, are illustrated in Fig. 3, in which $\theta=1$ and 0 correspond to the heated-plate temperature and the fluid temperature in free stream, respectively. It is observed in a) and b) of Figs. 2 and 3 that 1) mixing of the upper and lower streams of the plate downstream from the slot is stimulated, and 2) this increase induces the alternating change in the flow direction across the slot, resulting in amplification of the thermal diffusion from the plate along the flow. This change is suppressed by the flow acceleration due to a decrease in the channel width, as seen in Figs. $2 \mathrm{c}$ and $3 \mathrm{c}$. The alternating change in the flow direction across the slot yields an increase in thermal diffusion from the rear plate, which is affected by the blockage factor. This thermal behavior becomes clearer for heattransfer performance from the rear plate.

Figure 4 illustrates, for $R e=120$, the time history of the Nusselt number at one side wall of the rear plate. Note that numerical results during arbitrary time interval, that is, of $T=100 \mathrm{~s}$ to $110 \mathrm{~s}$ are depicted in the figure, because the thermal transport phenomenon,

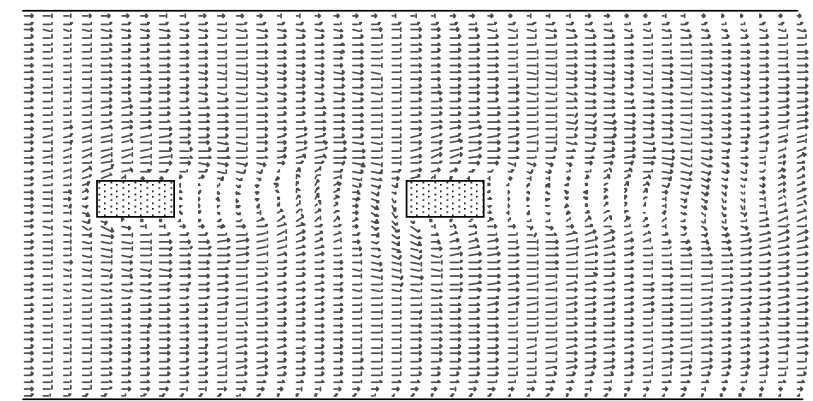

a) $\mathrm{k}_{\mathrm{q}}=\mathbf{0 . 1}$

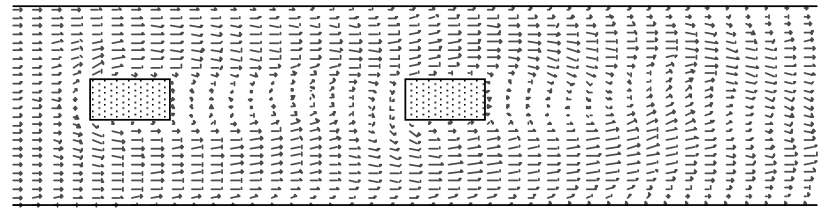

b) $\mathrm{k}_{\mathrm{q}}=0.2$

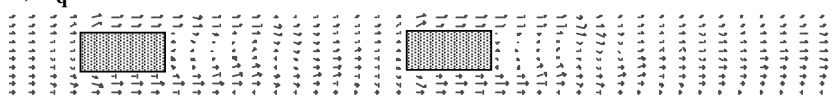

c) $\mathrm{k}_{\mathrm{q}}=\mathbf{0 . 5}$

Fig. 2 Instantaneous maps of velocity-vector field around the perforated plate for $R e=120$ and $T=100 \mathrm{~s}$.

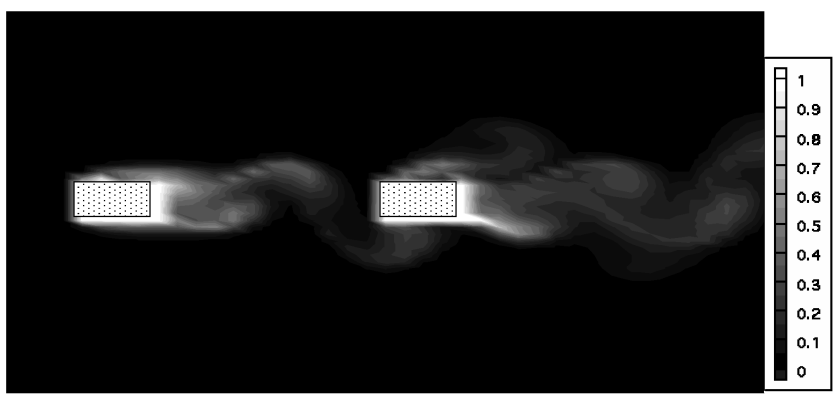

a) $\mathrm{k}_{\mathrm{q}}=\mathbf{0 . 1}$

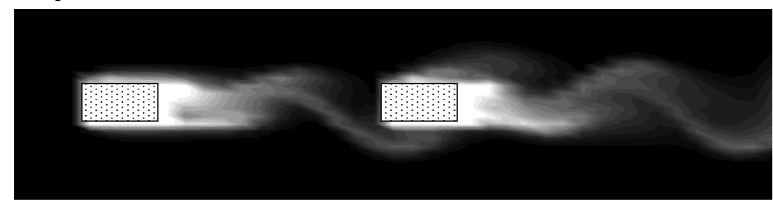

b) $\mathrm{k}_{\mathrm{q}}=\mathbf{0 . 2}$

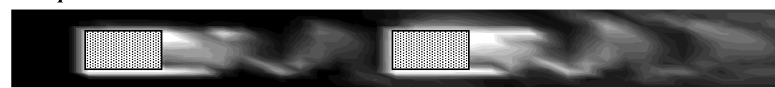

c) $\mathrm{k}_{\mathrm{q}}=\mathbf{0 . 5}$

Fig. 3 Instantaneous maps of temperature field around the perforated plate for $R e=120$ and $T=100 \mathrm{~s}$.

from the calculation results, becomes periodic after about $T=80 \mathrm{~s}$. Here, $\mathrm{Nu}$ implies the averaged Nusselt number over one side wall of the plate at each $t$. The oscillation of the heat-transfer rate with time is caused by the alternating changes in the fluid flow across the slot, as mentioned previously. This mechanism of heat-transfer enhancement is described in [23]. The Nusselt number is suppressed by a decrease in the channel width, that is, an increase in the blockage factor, because the alternating changes in the fluid flow across the slot is attenuated due to the presence of the channel walls. In contrast, heat-transfer rate is intensified in the narrow channel due to the substantial flow acceleration, in spite of the suppression of the alternating changes in the fluid flow. Furthermore, the oscillation of the heat-transfer rate with time disappears in comparison with the 


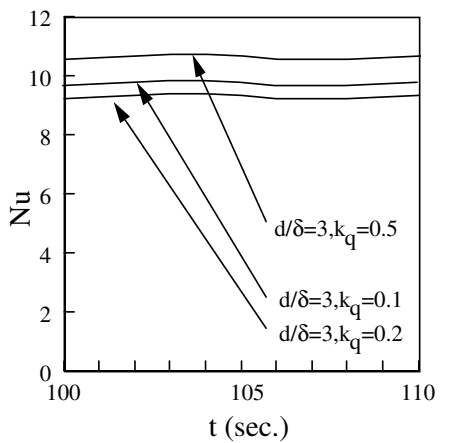

Fig. 4 Timewise variation of Nusselt numbers at rear plate for $\boldsymbol{R e}=120$.

wider channel flow case, as seen in Fig. 4. Heat-transfer characteristics become clearer for the timewise variation of flow patterns and isotherms over both sides of the single-slot-perforated plate.

Figures $\underline{5}$ and $\underline{6}$ depict the timewise variation of flow patterns over both sides of the single-slot-perforated plate. Consecutive images in Figs. $\underline{5}$ and $\underline{6}$ correspond to the numerical results for $k_{q}=0.2$ and $k_{q}=\overline{0} .5$, respectively, and are obtained at a time interval of $1.0 \mathrm{~s}$. Notice that velocity components are normalized by dividing by inlet velocity, as mentioned previously. Figure $\underline{6}$ shows a sequence of alternating crossing of flow streaklines from one side of the plate to the other through the slot. On the contrary, the timewise variation of the flow streaklines is substantially attenuated due to the narrow channel, as shown in Fig. 6. The corresponding temperature distributions, that is, isotherms as illustrated in Figs. 7 and 8. It is observed in Fig. 8 that the alternating change in the flow direction across the slot yields an increase in thermal diffusion from the rear plate. This results in a regular change in temperature distributions in the flow direction, particularly behind the rear plate. In contrast, such

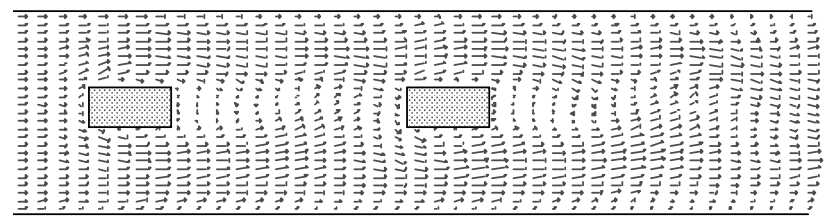

a)

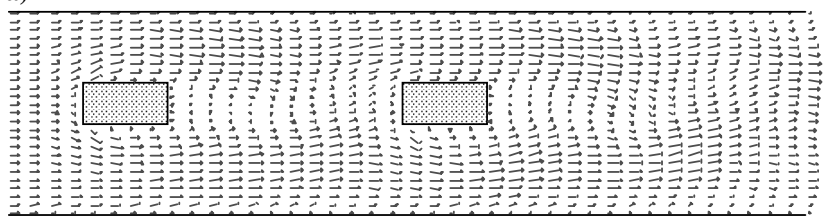

b)

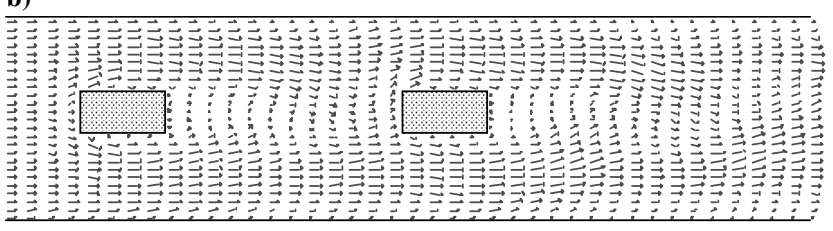

c)

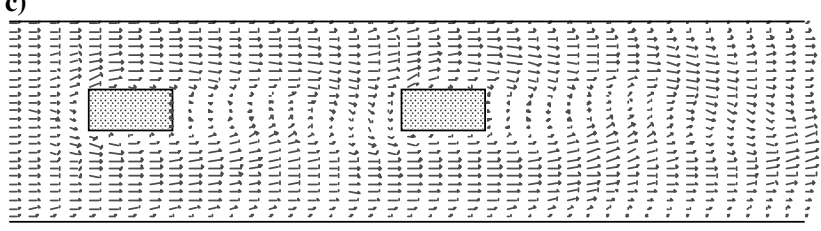

d)

Fig. 5 Timewise variation of velocity-vector field around the perforated plate at a time interval of $T=1.0 \mathrm{~s}$, for $k_{q}=0.2$ and $\operatorname{Re}=\mathbf{1 2 0}$.

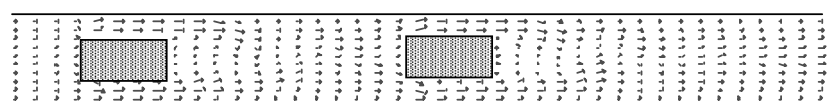

a)

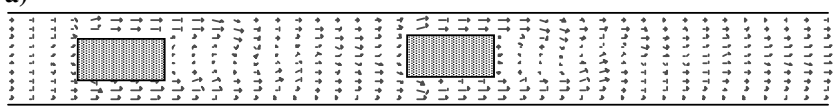

b)

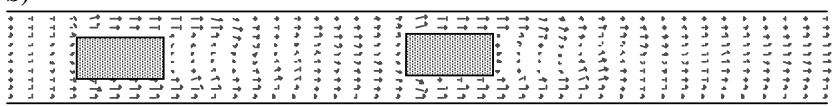

c)

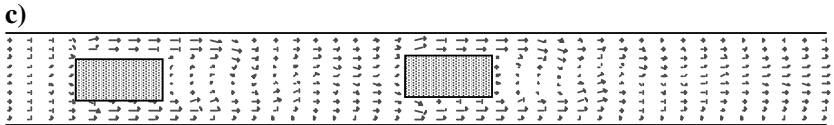

d)

Fig. 6 Timewise variation of velocity-vector field around the perforated plate at a time interval of $T=1.0 \mathrm{~s}$, for $k_{q}=0.5$ and $\operatorname{Re}=\mathbf{1 2 0}$.

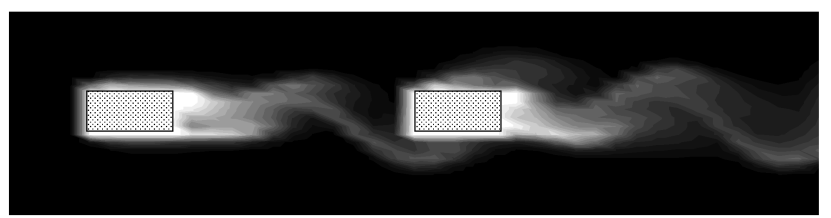

a)

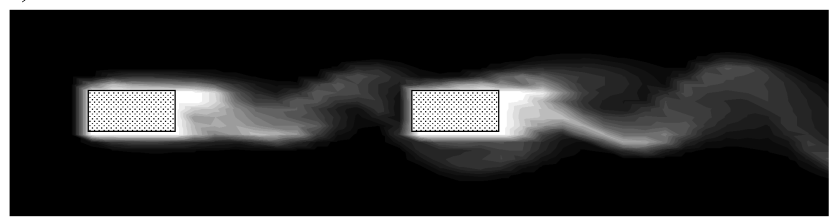

b)

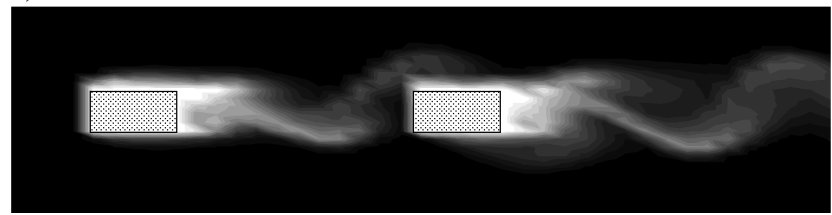

c)

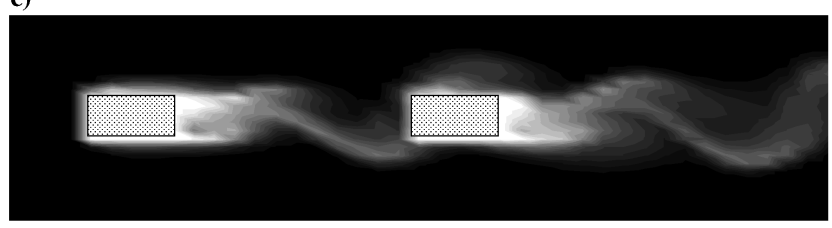

d)

Fig. 7 Timewise variation of temperature field around the perforated plate at a time interval of $T=1.0 \mathrm{~s}$, for $k_{q}=0.2$ and $R e=120$.

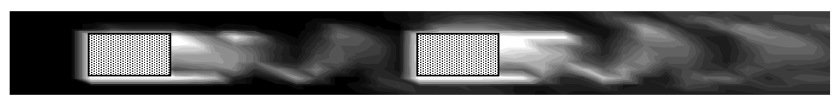

a)

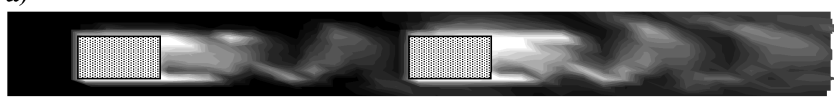

b)

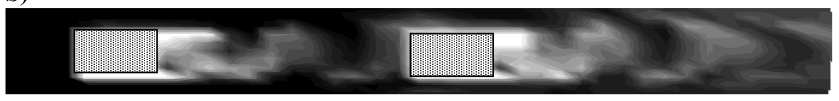

c)

Fig. 8 Timewise variation of temperature field around the perforated plate at a time interval of $T=1.0 \mathrm{~s}$, for $k_{q}=0.5$ and $R e=120$. 


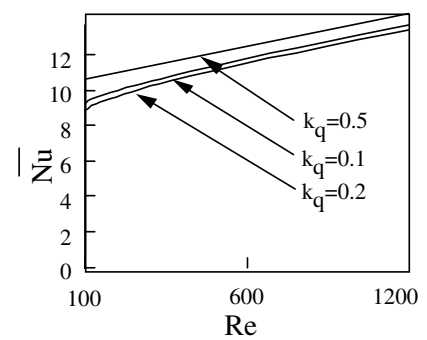

Fig. 9 Time-averaged Nusselt numbers for different blockage factor for $d / \delta=4$.

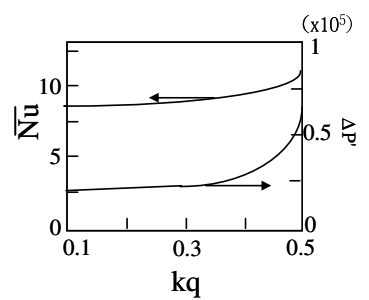

Fig. 10 Hydrodynamic and thermal performance for $R e=100$.

regular change occurs in the narrow channel flow (Fig. 8). It is found that 1) the alternating changes in the fluid flow, for $k_{q}=0.2$, result in an amplification of the heat-transfer performance at the rear wall, as seen in Fig. 4, and 2) further enhancement of heat-transfer rate yields in the narrow channel flow, that is, for $k_{q}=0.5$. This is because the flow is locally accelerated at the minimum cross-sectional area in the channel, so that the temperature gradient is substantially intensified in the vicinity of the heated plate.

The next task is to discuss the effect of the blockage factor on heattransfer performance from the slot-perforated plate. Figure 9 illustrates the time-averaged Nusselt number versus the Reynolds number with $k_{q}$, as a parameter. Although the Nusselt number is attenuated with an increase in the blockage factor, heat-transfer performance is intensified in the substantially narrow channel, that is, for $k_{q}=0.5$. This trend becomes larger in the lower Reynolds number region plotted. In other words, the effect of the blockage factor on heat-transfer performance becomes minor in the higher Reynolds number region. It is found that 1) as the channel width becomes narrow, an alternating change in the velocity and temperature fields is suppressed, resulting in deterioration of heattransfer performance, and 2) heat-transfer enhancement is stimulated, however, for a larger blockage factor due to local acceleration at the minimum cross-sectional area in the channel. Notice that for the larger blockage factor, the substantial pressure drop is needed to obtain the accelerated flow effect, that is, the pressure loss increases.

An attempt is made to obtain the optimum heat-transfer performance by taking the pumping power into account. Figure 10 , for $R e=100$, depicts the time-averaged Nusselt number on the plate fin against $k_{q}$ and the corresponding dimensionless pressure drop $\Delta P^{\prime}$ is superimposed in the same figure. It is observed that $\mathrm{Nu}$ increase with an increase in the blockage factor and the similar curve is shown for the pressure drop. In other words, the pressure drop is induced in the narrow space of the plate fins, as expected, and its trend is attenuated in the wider spacing. It is noted, for the $k_{q}$ range considered here, that the Nusselt number increases to $20 \%$, whereas the pressure drop amplifies to $240 \%$. This indicates that the optimum heat-transfer performance is obtained by suppressing an increase in the pressure drop, that is, higher thermal performance yields at $k_{q}=0.4$.

\section{Summary}

A numerical and experimental study has been performed on unsteady thermal-fluid flow over a perforated plate (consisting of two plates spaced at an interval of $d$ in the flow direction) installed in channels with different widths. Consideration was given to the roles of the Reynolds number and the blockage factor on the flow and temperature fields. It was found that:

1) Alternating changes in the fluid flow results in an amplification of the heat-transfer performance at the rear wall.

2) When the channel becomes narrower, the alternating change in the fluid flow is suppressed, resulting in an attenuation of the heattransfer performance at the rear wall of the rear plate.

3) Heat-transfer enhancement is stimulated for a larger blockage factor due to local acceleration at the minimum cross-sectional area in the channel, whose trend becomes larger in the lower Reynolds number region considered here.

References

[1] Wong, S., Duncan, J. D., Gibson, J. C., and Killackey, J. J., "Compact Condenser for Rankine Cycle Engine," Air Research Manufacturing Co., Final Rept. 71-7464, prepared for California Environmental Protection Agency, 1971.

[2] Kays, W. M., "The Heat Transfer and Flow Friction Characteristics of a Wave Fin, a Strip Fin, and a Perforated Fin Heat Transfer," Stanford Univ. Rept. 39, Dept. of Mechanical Engineering, Stanford, CA, 1958.

[3] Shah, R. H., and Osborn, H. H., Advanced Heat Exchanger Design of Compact Heat Exchanger When Operating in a Marine Environment, Final Rept., Air Preheat Company, Wellesville, New York, 1967.

[4] Liang, C. Y., and Yang, W.-J., "Heat Transfer and Friction Loss Performance of Perforated Heat Exchanger Surface," Journal of Heat Transfer, Ser. C, , Vol. 97, No. 1, 1975, pp. 9-15.

[5] Liang, C. Y., and Yang, W.-J., "Modified Single Blow Technique for Performance Evaluation on Heat Transfer Surface," Journal of Heat Transfer, Ser. C, , Vol. 97, No. 1, 1975, pp. 16-21.

[6] Liang, C. Y., Yang, W.-J., and Hung, Y. Y., "Perforated-Fin Type Compact Heat Exchangers for Gas Turbines," Proceedings of the Tokyo Joint Gas Turbine Congress, American Society of Mechanical Engineering, New York, 1977, pp. 77-85.

[7] Lee, C. P., and Yang, W.-J., "Augmentation of Convective Heat Transfer from High-Porosity Perforated Surfaces," Proceedings of Heat Mass Transfer, Vol. 2, American Society of Mechanical Engineering, New York, 1978, pp. 589-594.

[8] Fujii, M., Seshimo, S. S., and Yamanaka, G., "Heat Transfer and Pressure Drop of Perforated Surface Heat Exchanger with Passage Enlargement and Contraction," International Journal of Heat and Mass Transfer, Vol. 31, No. 1, 1988, pp. 135-142.

[9] Hwang, G. J., Wu, C. C., Lin, L. C., and Yang, W.-J., "Investigation of Flow Drag and Forced Convective Heat Transfer in Perforated Coolant Channels," Transport Phenomena in Combustion, Vol. 2, edited by S. H. Chan, Taylor \& Francis, 1996, pp. 1747-1758.

[10] Liang, C. Y., "Heat Transfer, Flow Friction, Noise and Vibration Studies of Perforated Surface," Ph.D. Dissertation, University of Michigan, Ann Arbor, MI, 1975.

[11] Liang, C. Y., Lee, C. P., and Yang, W.-J., "Visualization of Fluid Flow Past Perforated Surfaces," Proceedings of the 4th Japanese Symposium on Flow Visualization, Visualization Society of Japan, Tokyo, 1976, pp. 69-73.

[12] Torii, S., and Yang, W.-J., "Numerical Flow Visualization of a SlotPerforated Flat Surface in Free Stream," Canada Society of Mechanical Engineering, Vol. 1, 1998, pp. 177-184.

[13] Torii, S., and Yang, W.-J., "Flow over a Slot-Perforated Flat Surface Between Two Parallel Plates," Proceedings of 8th International Symposium of Flow Visualization, No. 006, Visualization Society of Italy, Sorrento, 1998, pp. 1-8.

[14] Torii, S., Yang, W.-J., and Umeda, S., "Flow Visualization and Transport Phenomena over a Slot-Perforated Flat Surface between Two Parallel Plates," Journal of Flow Visualization and Image Processing, Vol. 5, No. 2, 1998, pp. 63-80.

[15] Amon, C. H., and Mikic, B. B., "Spectral Element Simulations of Unsteady Forced Convective Heat Transfer: Application to Compact Heat Exchanger Geometries," Numerical Heat Transfer, Pt. A, Vol. 19, No. 1, 1991, pp. 1-19.

[16] Suzuki, K., Xi, G. N., Inaoka, K., and Hagiwara, Y., "Mechanism of Heat Transfer Enhancement due to Self-Sustained Oscillation for InLine Fin Array," International Journal of Heat and Mass Transfer, Vol. 37, Suppl. 1, March 1994, pp. 83-96.

[17] Nigen, J. S., and Amon, C. H., "Time-Dependent Conjugate Heat Transfer Characteristics of Self-Sustained Oscillatory Flows in a Grooved Channel," Journal of Fluids Engineering, Vol. 116, No. 3, 1994, pp. 499-507.

[18] Kim, S. H., and Anand, N. K., "Use of Slots to Enhance Forced 
Convective Cooling Between Channels with Surface-Mounted Heat Sources," Numerical Heat Transfer, Pt. A, Vol. 38, No. 1, 2000, pp. 121.

[19] Zukauskas, A., and Ziugzda, J., Heat Transfer of a Cylinder in Crossflow, Hemisphere Publishing Corporation, 1985.

[20] Harlow, F. H., and Welch, E. J., "Numerical Calculation of TimeDependent Viscous Incompressible Flow of Fluid with Free Surface," Physics of Fluids, Vol. 8, No. 12, 1965, pp. 2182-2189.

[21] Hirt, C. W., Nichols, B. D., and Romero, N. C., "SOLA-A Numerical
Solution Algorithm for Transient Fluid Flows," Los Alamos Science Laboratory, Rept. LA-5852, 1975.

[22] Torii, S., Yang, W.-J., and Umeda, S., "Flow Visualization and Transport Phenomena over a Slot-Perforated Flat Surface Between Two Parallel Plates," Journal of Flow Visualization and Image Processing, Vol. 5, No. 2, 1998, pp. 63-80.

[23] Torii, S., and Yang, W.-J., "Thermal Fluid Transport Phenomena over Slot-Perforated Flat Surface in Channels," Journal of Flow Visualization and Image Processing, Vol. 9, No. 4, 2002, pp. 281-285. 